\title{
Une approche de la culture professionnelle des soignants à travers les films de recrutement
}

An approach to the work culture of the health sector through recruitment films

\section{Caroline Benedetto}

\section{(2) OpenEdition}

1 Journals

Édition électronique

URL : http://journals.openedition.org/asp/5758

DOI : 10.4000/asp.5758

ISSN : 2108-6354

Éditeur

Groupe d'étude et de recherche en anglais de spécialité

\section{Édition imprimée}

Date de publication : 2 mars 2019

Pagination : 25-47

ISSN : 1246-8185

\section{Référence électronique}

Caroline Benedetto, « Une approche de la culture professionnelle des soignants à travers les films de recrutement », ASp [En ligne], 75 | 2019, mis en ligne le 02 mars 2020, consulté le 02 novembre 2020. URL : http://journals.openedition.org/asp/5758 ; DOI : https://doi.org/10.4000/asp.5758

Ce document a été généré automatiquement le 2 novembre 2020.

Tous droits réservés 


\title{
Une approche de la culture professionnelle des soignants à travers les films de recrutement
}

An approach to the work culture of the health sector through recruitment films

\author{
Caroline Benedetto
}

\section{Introduction}

1 Cette contribution, qui se situe dans le champ des études en anglais de spécialité, vise à caractériser un genre de discours ${ }^{1}$ professionnel : les films de recrutement produits par les organisations de santé britanniques.

2 Ces organisations sont des hôpitaux, des associations professionnelles responsables des spécialités, des autorités sanitaires régionales, ainsi que des organismes chargés d'informer les candidats sur le rôle, les compétences et les modalités d'exercice propres à chaque métier.

3 Nous avons envisagé leurs productions audiovisuelles comme des "publicités institutionnelles » (Lugrin 2006: 49), dont l'objectif principal n'est pas de vendre des produits (à la différence de la publicité mercantile), mais d'attirer et de retenir des professionnels en interne. Les films visent aussi à projeter, par l'intermédiaire des professionnels qui en sont les protagonistes, une image positive des organisations pour leur donner plus de crédibilité et de légitimité. C'est également en ces termes que les dictionnaires spécialisés en sciences de la communication et en sciences de gestion/ marketing définissent la notion d'institutional advertising: " an advertising message that promotes the image, reputation, and ideas of the company or organization, as opposed to the firm's product and services » (Govoni $2004: 102)^{2}$.

4 Les efforts réalisés par les organisations pour (re)valoriser leur image ne sont pas spécifiques au domaine de la santé britannique: ils sont observables dans d'autres milieux professionnels, comme ceux de l'ingénierie, du droit, de l'enseignement. Les 
films «\#ThisIsEngineering» (Royal Academy of Engineering 2018), «Every Lesson Shapes a Life » (Department for Education 2018) et « Solicitor Brand Campaign» (Law Society 2018) en constituent quelques exemples récents.

Diffusés sur plusieurs canaux de communication (télévision, radio, presse papier, internet et réseaux sociaux, etc.), les films de recrutement produits ces dernières années donnent généralement à voir d'authentiques praticiens ${ }^{3}$ dans des scènes tournées en «milieu professionnel réel » et non en «milieu professionnel représenté dans la fiction " (Petit 2004). Leurs auteurs s'attachent à souligner les avantages et l'utilité sociale des activités de travail exercées, à déconstruire les idées reçues sur les métiers, et à attirer une plus grande diversité de profils au sein des professions et des organisations, tout en accordant une place centrale aux usagers qui bénéficient des services proposés.

Dans un premier temps, nous prenons appui sur quelques exemples terminologiques apparus au début des années 2000 pour montrer que la communication publique des organisations britanniques s'inspire de plus en plus de la publicité, ce qui tend à brouiller les frontières entre ces deux formes de communication. Puis, à partir d'un corpus de films de recrutement produits par des organisations de santé du RoyaumeUni, nous nous interrogeons sur les modes de représentation de l'identité professionnelle dans le discours. Quelle image les locuteurs donnent-ils à voir d'euxmêmes et de leur profession, et quels procédés stylistiques et rhétoriques mobilisent-ils pour emporter l'adhésion de leur public ? L'article se clôt par une réflexion sur l'intérêt pédagogique que présente le film de recrutement pour l'enseignement de l'anglais de spécialité, dans sa dimension culturelle.

\section{De la publicité mercantile à la communication de recrutement : un brouillage des frontières}

Depuis le début des années 2000, pour construire une identité et une image distinctives de chaque organisation en tant qu'employeur (Viot \& Benraïss-Noailles 2014:62) et ainsi attirer plus facilement de nouvelles recrues, les organisations britanniques s'inspirent largement des stratégies et des concepts développés jusqu'à présent par les entreprises du secteur marchand (Barrow \& Mosley 2005 ; Lacka-Badura 2017). Par conséquent, le discours qu'elles produisent est marqué par de nombreux emprunts au marketing et à la publicité, comme le note $\mathrm{A}$. Wæraas à propos de la rhétorique institutionnelle et de ses mutations: "new management buzzwords such as branding, identity, and reputation management have appeared on the agenda of many public organizations» (2010: 527). Le tournant publicitaire que prend la communication de recrutement apparait d'ailleurs clairement dans les appellations qui lui sont attribuées par plusieurs auteurs anglophones (recruitment advertising [Ryan et al.] 2000 ; recruitment marketing, personnel marketing et employment marketing [Roberts 1997]; [Asprey 2005] ; [Price 2007]).

8 Parmi les exemples terminologiques qui nous paraissent les plus révélateurs de l'hybridation des discours institutionnels et publicitaires, l'expression employer brand, traduite en français par "marque employeur", a retenu notre attention. Apparue à la fin des années 1990, elle représente la somme des efforts consentis par une organisation pour montrer aux collaborateurs, présents et à venir, qu'elle est un 
environnement collectif agréable où il fait bon travailler (Lloyd 2002). Elle combine, par conséquent, un certain nombre d'éléments qui reflètent la philosophie adoptée par ses dirigeants et qui définissent l'identité de chaque structure : «it encompasses the firm's values, systems, policies, and behaviours toward the objectives of attracting, motivating, and retaining the firm's current and potential employees » (Dell \& Ainspan $2001: 10$ ).

D'autres expressions également empruntées à la publicité, comme celle d'employer brand, font désormais partie intégrante du vocabulaire de recrutement des institutions anglophones, y compris celles du domaine de la santé britannique. Nous en proposons une liste non exhaustive dans le tableau $1^{4}$, à titre d'illustration.

Tableau 1. Quelques expressions terminologiques empruntées au marketing et à la publicité

\begin{tabular}{|l|l|}
\hline $\begin{array}{l}\text { Discours du marketing et de la } \\
\text { publicité }\end{array}$ & Discours de recrutement des organisations \\
\hline brand ambassadors & employee ambassadors \\
\hline commercial brand & employee engagement \\
\hline customer engagement & employee loyalty \\
\hline customer loyalty & employee retention \\
\hline customer retention & Employee Value Proposition (EVP) \\
\hline Unique Selling Proposition (USP) & job advertisement \\
\hline product advertisement & recruitment advertising \\
\hline product advertising & $\begin{array}{l}\text { recruitment marketing / personnel marketing } \\
\text { employment marketing }\end{array}$ \\
\hline product marketing & talent management \\
\hline $\begin{array}{l}\text { customer reward and recognition } \\
\text { programmes }\end{array}$ & employee reward and recognition programmes \\
\hline
\end{tabular}

10 En élaborant leur propre marque, les organisations promettent à leurs collaborateurs une offre exceptionnelle en termes de ressources humaines qui les différencie positivement des employeurs existants, comme le suggère l'émergence de l'Employee Value Proposition (EVP). Directement empruntée à la stratégie de l'Unique Selling Proposition (USP) dans le domaine du marketing des produits, l'EVP désigne une "promesse d'emploi unique" adressée par une organisation à ses membres pour montrer qu'elle est un employeur de choix (Kapoor 2010). Elle regroupe tous les avantages matériels et symboliques associés aux postes proposés: convivialité du travail en équipe, possibilité d'aménager son emploi du temps, possibilités offertes aux candidats de continuer à se former en interne, d'évoluer dans la hiérarchie, etc. Toutefois, si les organisations se revendiquent comme des marques employeurs qui 
savent s'adapter aux différents besoins de leurs salariés, elles attendent également beaucoup d'eux en retour puisque, comme le rappelle un professionnel du recrutement, les avantages qui leur sont promis sont «une nouvelle façon d'aller chercher l'engagement et la productivité » de la force de travail (Girardeau 2018 : §4).

De plus, comme le font les enseignes du secteur marchand lorsqu'elles embauchent des ambassadeurs de marque ou des influenceurs (brand ambassadors) pour vanter les mérites d'un produit, les organisations publiques ou privées font appel à leurs employés pour améliorer leur image et leur réputation. Institués comme "salariésambassadeurs " (employee ambassadors), ils sont incités à exprimer personnellement leur soutien à l'organisation dont ils font partie, par exemple en communiquant de façon valorisante à propos de leur travail sur les réseaux sociaux numériques professionnels comme LinkedIn. L'intégration aboutie de cette expression dans le discours des organisations montre que, de la même façon que la notoriété d'une marque du secteur marchand se fonde sur l'implication de ses clients, la construction de la réputation des « marques employeurs » dépend étroitement de la collaboration de ses membres ${ }^{5}$. Ces derniers ont le pouvoir d'influencer la perception du grand public vis-à-vis d'une organisation (Chernatony 1999) en particulier grâce à la place qu'ils y occupent et à leur accès à l'information (Rao et al., 2001). Comme le souligne S. Pratt « as known, nobody is more influential, when it comes to communicating the company's brand, than the employees themselves » (2016: \$6). En d'autres termes, les membres de la société civile les considèrent comme légitimes et ils ont confiance en leur capacité à évaluer une organisation et à diffuser de l'information crédible (Stuart 2000).

Enfin, pour encourager la participation de leurs employés, les organisations britanniques se sont inspirées des dispositifs de reconnaissance et de récompense lancés aux États-Unis il y a plus d'un siècle (employee reward and recognition programmes), en leur offrant un certain nombre de rétributions, comme des mentions honorifiques, des remerciements ou des prix. Dans le domaine de la santé, par exemple, les programmes NHS Heroes et Extraordinary People Award invitent les membres du personnel soignant et les patients à désigner un professionnel ou une équipe de professionnels particulièrement méritants. Ainsi, dans les organisations du National Health Service (NHS), des nominations sont proposées dans plusieurs catégories, allant de Top-quality Patient Care à Lifetime Achievement Award, en passant par Excellence in Values and Behaviours et Healthcare Team of the Year ${ }^{6}$. L'enjeu de ces dispositifs est non seulement d'encourager l'ensemble des employés à améliorer leur rendement et leur efficacité, mais aussi de diffuser, à travers la médiatisation de ceux qui affichent un comportement exemplaire, une image valorisante des organisations de santé auprès du grand public.

\section{Décryptage d'un genre publicitaire institutionnel : le film de recrutement}

\subsection{Caractéristiques du corpus et méthodologie}

Le corpus qui a nourri cette étude est constitué de onze films au format court (de 1,30 à 8,41 minutes) que nous avons répertoriés au sein d'un tableau, puis classés en fonction des trois critères suivants : la profession ou la spécialité mise en avant, le titre du film, suivi de son adresse URL et de sa date de mise en ligne sur internet (cf. annexe 1). Dans 
le présent article, nous avons désigné chaque film par des formes abrégées correspondant à chaque profession (GM pour general medicine, $\mathrm{P}$ pour psychiatry et $\mathrm{N}$ pour nursing). Un chiffre leur a été associé pour permettre au lecteur de mieux les identifier.

Traditionnellement, les films sont diffusés en premier lieu sur le site internet des organisations, des "vitrines institutionnelles» (Barats 2009) qui permettent aux internautes de comprendre le fonctionnement des structures, tout en garantissant à ces dernières une certaine visibilité, attractivité et transparence vis-à-vis de leurs salariés et du grand public ${ }^{7}$. Ils sont ensuite publiés sur les réseaux sociaux numériques tels que Youtube, Twitter, Facebook et LinkedIn, avant de faire l'objet de partages et/ou de commentaires publiés sur la toile.

Les paroles et les textes affichés à l'écran ont été retranscrits manuellement à l'aide du logiciel de traitement de texte Microsoft Word, ce qui nous a permis d'obtenir un corpus de 9000 mots. Nous avons analysé chaque film en prêtant attention au message linguistique et aux aspects audiovisuels. Ainsi, nous avons examiné non seulement le contenu de chaque production (avec son sujet et ses protagonistes), mais aussi la manière dont ce contenu est mis en scène, notamment grâce au cadrage, aux sons, à l'agencement, aux images, au recours à une voix off et/ou à des sous-titres, à la présence de musiques, ainsi qu'au rythme de défilement des images. L'analyse de ces différentes dimensions nous a permis de mieux saisir de quelle façon le réel professionnel est donné à voir et à entendre.

\subsection{Un discours produit en contexte de pénurie}

Les commanditaires des films sont des organisations de santé relevant du National Health Service (NHS), qui est l'un des principaux employeurs du Royaume-Uni et le cinquième le plus important dans le monde, réunissant 1205949 salariés en janvier 2018 selon les études statistiques sur l'emploi publiées par le gouvernement britannique ${ }^{8}$.

17 Contribuant largement à l'organisation des soins et à leur prise en charge, cet organisme connaît, depuis la fin des années 1990, une importante pénurie de personnel soignant, dont la presse britannique (généraliste et professionnelle) se fait souvent l'écho (The Guardian 2018; BBC 2017, 2018a, 2018b ; RCN 2018; BMJ 2018). De graves dysfonctionnements sont signalés dans les hôpitaux, où les praticiens sont surchargés de travail et où les listes d'attentes de patients se révèlent de plus en plus longues. La pénurie est liée à d'importantes restrictions budgétaires émanant du gouvernement britannique, au vieillissement des patients, aux départs de nombreux praticiens (à la retraite ou bien à l'étranger dans l'espoir d'y trouver de meilleures conditions de travail). Elle concerne plusieurs corps professionnels, notamment les médecins urgentistes, les chirurgiens, les sages-femmes et les infirmiers ${ }^{9}$.

Pour tenter de résoudre cette crise humaine et professionnelle, le NHS fait appel à un grand nombre de praticiens au-delà de ses frontières nationales, comme en témoigne un rapport publié en 2017 par le régulateur de la profession médicale, le General Medical Council, signalant qu'un tiers des médecins qui exercent leur activité en contexte britannique ont été formés dans d'autres pays, essentiellement non européens, comme l'Inde, le Pakistan et l'Afrique du Sud (GMC 2017 : 44). Bien que ces praticiens doivent justifier d'un certain niveau de langue anglaise pour être autorisés à exercer leur 
activité, ils peuvent avoir des difficultés à intégrer les normes socioculturelles et socioprofessionnelles en vigueur dans leur pays d'accueil, comme l'ont montré A. Slowther et al. dans leur étude sur l'acculturation des médecins néo-arrivants au Royaume-Uni $(2012: 105)^{10}$.

\subsection{Les professionnels définis comme " cœur de cible »}

19 En conformité avec le modèle générique établi par V. Bhatia qui identifie les principaux mouvements rhétoriques des publicités traditionnelles (2005: 214), le film de recrutement est généralement doté d'un titre spécifique ("headlines»), qui sert de

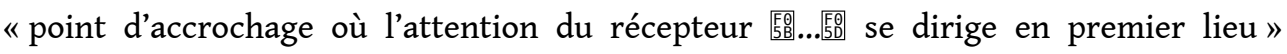
(Hoek 1981 : 248). Les termes qui le constituent, tels que «Choose Psychiatry. A Career That Makes A Difference» (P5) et "One Career, Endless Opportunies. Choose GP» (GM2), interpellent particulièrement les professionnels de santé.

De la même façon, les slogans qui accompagnent le nom et le logo de chaque employeur («signature line and logo", Bhatia 2005: 214) prennent la forme de messages personnalisés adressés aux futurs soignants. Ainsi, la devise «Train. Work. Live.» vante aux infirmiers du Pays de Galles (NHS Wales) la possibilité d'articuler aisément leur vie professionnelle avec leur vie personnelle (work-life balance) (cf. annexe 2 et film N10).

21 Les experts émettent aussi parfois des recommandations à l'attention des candidats comme "I would certainly encourage nurses to come and work for Bolton, especially in the community» (N9). Dans le discours, ces marques de soutien apportées aux organisations (« endorsement or testimonials »,que selon V. Bhatia [2005: 214]) contribuent à renforcer l'efficacité de la persuasion.

Enfin, comme bon nombre de publicités contemporaines, le film de recrutement se clôt par un "appel à l'action", suivant le mouvement rhétorique "soliciting response " (ibid.). Au moyen d'une interrogation ou d'une injonction impérative telle que " Interested in a career in health? Visit www.healthcareers.nhs.uk» (GM1, P4) ou "We are recruiting now. Search nursing careers" (N11), renforcée par l'affichage de mots-dièses (hashtags) comme \#chooseGP (GM2) et \#YouCanBeThatNurse (forme identifiée sur Twitter), les collaborateurs potentiels sont invités à consulter les sites web des organisations pour obtenir des renseignements complémentaires et y déposer leur dossier de candidature.

Ces mécanismes d'accroche et de persuasion montrent que le film de recrutement est une communication sur mesure qui a pour cible principale les professionnels de santé. Cette tendance au ciblage et à la personnalisation est d'ailleurs confirmée par les recommandations portées à l'attention des recruteurs du NHS : «it's important to think about your audience and tailor your adverts to suit. For example, the ambulance services often use radio advertising to target their audience who are typically out on the road" (NHS Employers 2018: 28). Dans le but d'inciter les jeunes générations à rejoindre les organisations de santé, les films de recrutement sont aussi largement diffusés sur les réseaux sociaux numériques.

\subsection{Une proximité et une familiarité avec un public élargi}

Bien que les films de recrutement forment un discours élaboré en priorité par et $\grave{a}$ destination de spécialistes du domaine de la santé, le vocabulaire employé n'est 
résolument pas technique. En effet, notre corpus contient moins de dix termes qui relèvent du champ spécialisé de la santé parmi lesquels figurent deux références à des spécialités médicales complémentaires: eating disorder psychiatry (les troubles du comportement alimentaire) et substance misuse psychiatry (l'addictologie) (P4).

Nous n'y avons repéré que deux sigles qui pourraient être inconnus des profanes car ils désignent des noms de professions (OT pour occupational therapist, dont l'équivalent français est ergothérapeute) (GM2), ainsi que des états du patient (PTSD pour posttraumatic stress disorder, un état de stress post-traumatique) (P5). Cette faible siglaison est donc plutôt surprenante étant donné que l'anglais de la santé comporte habituellement une profusion d'abréviations, ce qui en fait une langue codifiée, difficilement accessible aux non-initiés (Beltran-Vidal 2007; Faure 2010; Carnet et al. 2017).

Les rares expressions spécialisées que nous avons repérées au sein du corpus font l'objet des stratégies classiques de vulgarisation, telles que la reformulation, la définition ou l'explicitation, comme dans les passages suivants où les locuteurs s'attachent à décrire leur rôle et à définir une pratique professionnelle pouvant être inconnue du grand public :

[1] Mark Clement, Advanced Nurse Practitioner. My role is varied, I work 70\% clinical, which means that I'm out and delivering care and $30 \%$ of my time will be nonclinical, where I may be involved in education and research. (N8)

Rebecca Jenkinson, General Practitioner. My week can be really varied. I do six sessions, so 3 days a week as a general practitioner and during those days I will be seeing patients. (GM1)

Sr. Julie Roye, Advanced Nurse Practitioner. What I do with the patients is something called motivational interviewing ${ }^{11}$ - it's the patient opening up about how they feel about their health and what I can do with them. (N8)

Les efforts de clarification réalisés par les locuteurs ne visent pas seulement à rendre les propos des experts accessibles aux non-spécialistes : ils font aussi partie intégrante de la démarche de persuasion puisque, comme le rappelle $\mathrm{C}$. Beaudet à propos de la communication institutionnelle, la clarté a valeur d'argument (2003 : \$58). Ce principe est d'ailleurs explicitement mentionné dans les recommandations adressées aux recruteurs du NHS: «providing clear, accurate and accessible information for prospective candidates is essential if you are to make them feel supported and positive about applying to work for you » (NHS Employers 2014 : \$3). Le choix de recourir à un langage proche de celui du citoyen ordinaire crée ainsi un rapprochement entre les employeurs et les candidats. Il permet aussi aux locuteurs de se rapprocher du grand public, en construisant l'image de professionnels accessibles, bons communicants et proches de leurs patients.

L'impression de proximité se produit également dans les nombreuses marques d'oralité qui ponctuent les films de recrutement. Les énoncés sont brefs et marqués par un haut degré de simplicité, tant lexicale que syntaxique :

[2] It's not a normal job. We're looking for veins, keeping the music playing, we say hello, we wave goodbye, we're always here or we're always there. (N11)

La plupart d'entre eux relèvent des registres courants et familiers, et ils contiennent des expressions idiomatiques, comme dans les exemples suivants :

[3] [Doctor]. Being a GP is very varied. [...]. Every time I call a patient in, the clinical presentation can differ, and that keeps you interested, it also keeps you on your toes. (GM2) 
[Nurse]. We take a reading, agree a plan, make a cuppa. (N11) verbes à particule (phrasal verbs), caractéristiques d'un langage parlé, plutôt relâché, qui s'utilise habituellement avec des proches ou des collègues de travail :

[4] [Director, NHS East of England]. it'll be a terrific and rewarding career. (N6)

[Nurse]. I know, for sure, that I've helped many patients. (N8)

[Doctor]. I turn up about one hour before the match and check in with the players. (GM1)

[Patient]. I left the Armed Forces after 20 years and developed PTSD. It was completely unpleasant. I was completely on my own. Right on the edge. [...] She said about putting me right from day one. (P5)

L'impression de proximité est rendue également sur le plan visuel, dans les représentations de collègues souriants, réunis autour d'un canapé ou d'une tasse de thé, qui évoquent une ambiance conviviale voire familiale (cf. annexes 3 et 4). Les spectateurs des films accèdent ainsi à un cadre harmonieux, propice à la collaboration entre acteurs, et où les frontières entre les espaces professionnel et personnel tendent à s'estomper, comme on peut le voir dans la scène qui dépeint l'étreinte chaleureuse entre une infirmière et sa patiente (cf. annexe 5), véhiculant l'idée d'«extimité " (Tisseron 2011) selon laquelle l'intimité, extériorisée, est donnée à voir dans l'espace public afin de consolider et de valoriser l'image de l'énonciateur. de travail idyllique, exempt de contestations :

[5] My colleagues are lovely, we get along very well, it's like a family. (N9)

I did a placement here, I knew that I wanted to work here when I qualified. It's such a nice place to work. Everyone's bubbly, cheerful, so smiling. (N9)

I remember when I came here first I was overwhelmed just by the friendliness of people. (N9)

The 'plus' points when you get to really work with someone who's been struggling. Being able to be present in people's lives and provide that continuity of care and, in some cases, friendship. (GM1)

Le choix des pronoms personnels, visant à personnaliser le "message publicitaire » adressé à chaque cible (les professionnels ou le grand public), donne également une impression de proximité aux co-énonciateurs afin d'emporter plus facilement leur adhésion. C'est le cas du "you d'adresse », qui désigne tantôt les futurs collaborateurs (dans la première proposition), tantôt les usagers des services de soins (dans la seconde proposition) :

[6] [Bolton Hospital]. It's somewhere you'd actually want to work and somewhere you wouldn't mind being looked after. (N9)

Par ailleurs, la présence du pronom « we inclusif » (Skelton et al. 2002: 485), qui intègre les profanes dans le discours des experts et réciproquement, semble favoriser les échanges et permettre plus facilement qu'un dialogue se noue entre acteurs :

[7] We are Michelle, James, Garry, Ivan, Adam and Frankie and at 3:49 this morning we were Maisie too - seven pounds two ounces - all doing well. We are the NHS. (N11)

We don't do it for the thank you's. We do it for Janis, Alfie. For Jack. For all of us. (N11)

L'établissement d'une plus grande proximité avec les co-énonciateurs s'exprime enfin dans les jeux de connotations, notamment dans l'emploi de «look after». Si, dans son sens premier, ce verbe à particule s'applique à des sujets théoriquement vulnérables, 
dont il faut protéger les intérêts, comme les patients (« to keep someone healthy, safe, or in good condition " selon le dictionnaire Collins); dans notre corpus, il caractérise également les professionnels de santé, véhiculant l'idée que les organisations se soucient autant du bien-être des patients que de celui de leurs salariés. Dans les extraits ci-dessous, qui proviennent du même film, look after renvoie premièrement aux patients et à leurs proches, puis il prend la forme d'un participe passé adjectival qui décrit le ressenti du personnel soignant :

[8] To be able to care for these people who are such in a vulnerable position, just look after them and their families when they're with them. It's just an absolute privilege. (N9)

There's a really good, supportive, nurturing environment, and you do tend to leave your shift feeling nurtured and looked after. (N9)

En somme, à la manière des annonceurs publicitaires qui instaurent une relation étroite entre la marque et le public ciblé à des fins de captation et de persuasion, les protagonistes des films légitiment leur opinion en adoptant un style de communication accessible, direct et efficace qui favorise les rapprochements et les échanges entre acteurs. Ce choix stratégique marque une rupture, d'une part avec les discours spécialisés intra-professionnels du domaine de la santé qui sont souvent codifiés, et d'autre part avec les communications formatées et parfois impersonnelles qui émanent des institutions.

\subsection{Miroir social ou fiction de l'ordinaire?}

Les films de recrutement, comme nous l'avons vu au début de cet article, font apparaître des soignants qui interagissent avec leurs patients et/ou avec leurs confrères dans leur environnement quotidien de travail, ce qui donne à leur discours un certain réalisme. Les scènes du film "We are the NHS " (N11), notamment, ont été filmées in vivo, au moment de leur déroulement à l'hôpital, en raison des délais très courts impartis à l'équipe de production, comme le confie son réalisateur à la presse :

[9] It was a terrifying pace, but it turned out to be one of the greatest strengths of the film [...]. Everything is normally so planned that it can drain all of the life out of a film. Because we couldn't do that for this, everything was real and captured ad hoc, and it gave the film vitality. (Kiefer $2018:$ §12)

L'auteur ajoute que cette configuration particulière lui a permis de capturer des moments où la réalité dépasse la fiction, comme la danse proposée par des infirmières à leur patient atteint d'un cancer, ou l'accouchement par césarienne d'une mère ayant préalablement donné son accord pour que la scène soit filmée et portée à l'écran (ibid. : \$13).

Toutefois, les représentations du réel professionnel proposées dans les films du corpus semblent altérées par un recours au pathos et à la dramatisation puisque, comme dans toute publicité, «ce qui se donne à voir socialement n'est pas seulement diffusé, mais hypertrophié » (Sacriste 2001: \$23). Certains des films que nous avons examinés, en effet, narrent des "récits dramatisants dans lesquels sont mis en avant victimes et héros afin de produire tantôt de l'angoisse, tantôt de l'exaltation » (Charaudeau 2009 : 9). Ainsi, dans le film «Choose Psychiatry. A Career that Makes A Difference » (P5), des effets de contraste sont marqués dans le champ visuel par des plans rapprochés de la caméra qui font d'abord apparaître les visages tantôt angoissés tantôt désespérés des patients, puis les expressions sereines et confiantes des professionnels. Visant à 
provoquer des émotions plus ou moins fortes chez le spectateur, ce contraste entre soignés et soignants est accentué par une série d'adjectifs qui expriment les sentiments de mal-être et/ou de détresse psychologique dont souffrent les premiers ( $I$ I was totally trapped", "anxious", "depressed", "beaten up by the birth", "sleep-deprived, hallucinating, and psychotic", par exemple) et ensuite par des verbes d'action qui soulignent l'impact décisif de l'aide apportée par les seconds ("she kept me alive and kept my family together » ou " she gave me the support I needed to stay and fight. It's because of her that I'm still here today» [P5]).

En outre, la proximité et la familiarité de ton qui se dégagent des films de recrutement ne sont qu'apparentes. Les protagonistes sont soigneusement choisis par les organisations en fonction de "leur aptitude à passer auprès des médias " (Riou 1999: 16) et leurs échanges, à la différence d'une conversation spontanée, sont préparés à l'avance. Les questions posées aux experts contribuent à délimiter les tours de paroles et à orienter leur discours ${ }^{12}$, ce qui n'aboutit qu'à « une illusion d'oralité » (Fowler 1991) et « de familiarité » (Fairclough 1995). Formant un discours particulièrement subjectif, les films proposent des descriptions toujours valorisantes du travail proposé en ne laissant aucune place aux points de vue divergents. On n'y relève presque aucune référence au stress que représente le travail à l'hôpital ou aux obligations d'efficacité et de performance auxquelles sont quotidiennement soumis les professionnels de santé dans le cadre de leur activité (Benedetto 2017 : 140). Lorsque des défis sont évoqués, c'est pour montrer qu'ils ont été surmontés, notamment grâce à l'entraide et à la solidarité qui unit les membres du personnel soignant, suivant les propos de cette sagefemme: "it can at times be very difficult but there's excellent support here. We have supervisors of midwives to support staff through any difficult experiences that they may be having ${ }^{(\mathrm{N} 9)^{13}}$.

Les représentations idylliques que donnent à voir ces «fictions de l'ordinaire " (Soulages 2013) peuvent donc surprendre le spectateur, compte tenu du contexte de crise humaine et sociale que traverse actuellement le système de santé britannique. Elles semblent indiquer une tendance, de la part des locuteurs, à feutrer ou à gommer les réalités de terrain pour les rendre plus acceptables. Il s'agit d'un procédé rhétorique privilégié par les annonceurs publicitaires, mais aussi d'une stratégie de «lissage discursif » adoptée par les institutions (Oger \& Ollivier-Yanniv 2006), et qu'il n'est pas toujours aisé de mettre en œuvre, comme le remarque L. Powell dans son analyse des campagnes médiatiques menées en contexte militaire américain :

[10] Recruitment films are an art, as their makers have to find the right balance of adventure- without looking like a boring or tedious job. They lure individuals with promises of an exciting life without ever informing them of the other side of reality. (2014:174)

Il appartient donc aux organisations de veiller à ne pas donner une vision mensongère de l'environnement professionnel et des postes proposés, et d'apporter aux salariés des preuves tangibles de leur engagement pour ne pas perdre de leur légitimité et de leur crédibilité.

\subsection{Le film de recrutement : un vecteur de normes professionnelles}

43 Si par l'accessibilité de ses messages, l'informalité de son style, et son inscription dans la subjectivité, le film de recrutement s'apparente aux formes publicitaires 
traditionnelles, c'est en tant que vecteur de normes professionnelles qu'il se singularise.

Les films de recrutement que nous avons analysés revêtent l'apparence, certes plus discrète que les aspects argumentatifs qui viennent d'être décrits, mais néanmoins perceptible, d'un discours normatif ou de régulation qui cherche à imposer des lignes de conduite internes et externes aux membres de l'organisation, comme l'ont montré C. Oger et C. Ollivier-Yanniv à propos des campagnes de recrutement émanant des institutions (2006: \$35). Une lecture attentive de la transcription des films montre effectivement que les locuteurs participent, de façon insidieuse, à une régulation des comportements professionnels et des "savoir-être ", perceptible dans les références aux attributs requis pour l'exercice du métier :

[11] Mark Clement. Advanced Nurse Practitioner. I think adaptability is a key really. I would hope that competence is key too, but you have to be adaptable. (N8)

Jane Kay. Ward Manager. Coronary Care Unit. Teamwork is imperative. [...]. We communicate with patients and families on a daily basis. (N9)

Trish Armstrong-Child, Director of Nursing. Over the past twenty years there's been lots of changes to the nursing world, and lots of advanced practice but fundamentally the nursing model remains the same, it's about people who want to be kind and caring and compassionate, and that's the basis of what we're looking for for our nurses coming in. (N9)

Dans le premier exemple cité, comme dans beaucoup d'autres passages du corpus, le recours au pronom personnel you d'adresse donne d'ailleurs au texte la forme d'un contrat moral auquel tout professionnel de santé doit adhérer : « using 'you' to make it clear that each statement applies not just to a hypothetical or 'other' doctor, but to each doctor who reads it », comme le notent $\mathrm{A}$. Chisholm et $\mathrm{J}$. Askham à propos des guides de bonnes pratiques élaborés au Royaume-Uni (2006:31).

Nous avons aussi relevé un certain nombre de verbes d'action tels que assess, diagnose, evaluate, prescribe, treat, supervise and support, qui sont prononcés par une voix hors champ (« voix off ») ou qui apparaissent à l'écran afin de décrire le travail à l'hôpital (N8). Identifiés dans d'autres discours managériaux comme les référentiels de compétence et les codes de conduite, ces verbes servent moins, nous semble-t-il, à renseigner les soignants sur le déroulement des tâches qui leur seront confiées qu'à mettre en avant la performance et le professionnalisme qui sont exigés d'eux par les organisations, et dont l'importance nous parait renforcée par le défilement rapide des images.

47 À la différence d'autres discours de régulation comme les guides de bonnes pratiques, ponctués d'auxiliaires de modalité qui expriment explicitement la contrainte, nous n'avons toutefois relevé en corpus qu'une seule occurrence de must et quelques rares mentions de have to, notamment dans "you have to have those difficult conversations with the family" dans le discours d'une cadre de santé, qui souligne l'importance des aptitudes de communication des soignants (N9). Ainsi on peut se demander si le discours de recrutement, dès lors qu'il assure très activement la promotion de l'image des professions de santé à des fins de persuasion et de captation, ne cherche pas à masquer l'existence des normes et des rapports hiérarchiques qui sont pourtant inhérents à toute organisation et qui se font de plus en plus explicites dans le domaine de la santé britannique (Benedetto 2017 : 251-255).

$\mathrm{Au}$ sein du corpus, on perçoit toutefois la présence de ces normes - en particulier celles qui sont portées par la nouvelle gestion publique (New Public Management): les 
soignants sont incités à donner le meilleur d'eux-mêmes ( $I$ I really believe that Bolton offers nurses the potential to be the best they can be ", N9), à veiller continuellement à la sûreté des soins ( recently we won an award for Best Breast Cancer Care in the Greater Manchester area and that's for the safety culture, so we're really proud of that ", N9), à se former tout au long de leur carrière au nom d'une amélioration continue de la qualité des services proposés aux usagers ( I I think you build on the blocks of your nursing as being caring, compassionate, being a patient's advocate, understanding how vulnerable they are, but then with the additional skills, you can step up to care for them ", N8), et à faire preuve d'une plus grande polyvalence ( $"$ in the end, we're going to want to have a highly trained, highly competent consultant work body who are able to do the same missions of clinical work, teaching and research as all of our colleagues in every other speciality", P3). L'accent est mis, également, sur leur flexibilité et sur leur réactivité, comme l'indique cette infirmière :

[12] [T]he key skills for help are excellent communication, a real passion to work with children and families and make a difference for them, to be flexible and adaptable to the needs of the local community that you work in, and to be able to respond quickly when people have problems. (N7)

Ainsi le film de recrutement présente des dissemblances avec le discours publicitaire en ce qu'il contribue, en filigrane, à une régulation des comportements.

Les salariés-ambassadeurs qui figurent dans les films présentent, certes, de façon cohérente et vraisemblable leur parcours, leurs réalisations et leurs projets, mais ils affichent aussi et surtout diligemment leur conformité avec les attentes et les valeurs de l'organisation. L'enjeu est de créer et de diffuser un ensemble d'archétypes qui doivent susciter identification et appropriation de la part des futurs membres des futurs salariés. L'une des fonctions du récit ou storytelling, comme le note C. Salmon, consiste d'ailleurs à mettre en place « des engrenages narratifs, suivant lesquels les individus sont conduits à s'identifier à des modèles et à se conformer à des protocoles " (2007: 16). Les représentations nouvellement créées doivent améliorer l'efficacité persuasive de ces productions audiovisuelles, dont le but est autant de «faire voir » et de "faire croire » que de "faire adhérer " (Charaudeau 2009:3) leurs destinataires à la vision adoptée par les dirigeants de chaque organisation.

\section{Remarques conclusives sur les applications pédagogiques potentielles des films de recrutement}

Contrairement à la fiction à substrat professionnel (Petit 1999) ${ }^{14}$, qui dépeint de façon assez exhaustive un domaine spécialisé et ses discours, le film de recrutement ne donne pas à voir les formes langagières qui sont échangées par les soignants lorsqu'ils communiquent entre eux et qu'ils ne sont pas dans une posture de représentation visà-vis de l'extérieur. Par conséquent, il peut difficilement déboucher sur une étude des variétés spécialisées de la langue dans le cadre des enseignements LANSAD.

En revanche, ce matériau authentique mériterait d'être exploité sur le plan pédagogique car il donne accès à la culture professionnelle des soignants britanniques ${ }^{15}$, une tâche qui incombe à l'enseignant de langue de spécialité, « appelé à construire une démarche pédagogique permettant l'acquisition parallèle des compétences langagières et culturelles professionnelles » (Isani $2001: 106)$.

Bien que les récits individuels des professionnels s'enchevêtrent avec le discours promotionnel des organisations, ils apportent un éclairage précieux sur le contexte de 
production de leurs activités. Les protagonistes y transmettent un certain nombre d'informations non seulement sur leurs trajectoires individuelles mais aussi sur les modes traditionnels de professionnalisation des soignants (description des voies d'accès à chaque métier, des modalités d'exercice, des routines professionnelles, des spécialisations possibles, etc.), y compris sur les nouvelles normes qui s'appliquent à leur travail, comme nous l'avons vu dans la section précédente. Les films sont également utiles pour sensibiliser les apprenants aux éléments constitutifs de la culture professionnelle des soignants britanniques. L'étude de certains concepts spécifiques à cette communauté (et quelquefois, plus généralement, à celle des soignants anglophones) permettront à l'enseignant d'introduire des comparaisons culturelles avec le système français. Par exemple, l'observation des formes du mot care présentes à maintes reprises dans le corpus - mentionnées en tant que telles ${ }^{16}$ ou exprimées par des métaphores ${ }^{17}$ - pourra donner lieu à une étude plus approfondie de cette notion, dont la traduction en français ne recouvre pas les mêmes réalités qu'en anglais ${ }^{18}$. On pourra également examiner le concept d'empowerment ${ }^{19}$, un principe fondamental à la pratique des soignants anglophones qui figure très clairement parmi leurs attributions, y compris dans les films du corpus ${ }^{20}$.

Ces deux notions, relativement anciennes et courantes dans les pays anglophones mais qui se sont imposées tardivement en France ${ }^{21}$, pourront amener les apprenants à s'interroger sur le rôle et sur la place des soignants dans les deux systèmes de santé, français et britannique, et plus largement au sein de la société. En ce sens, le film de recrutement s'apparente à un outil réflexif qui leur donne la possibilité non seulement de se faire une idée plus précise de la profession envisagée (et de ce qu'elle requiert comme connaissances, compétences et dispositions), mais aussi de vérifier que leurs valeurs et leurs aspirations sont compatibles avec celles des organisations qu'ils souhaitent rejoindre.

\section{BIBLIOGRAPHIE}

ASPREY, Sarah. 2005. « Recruitment advertising ». In MACKAY, A. R. (dir.), The Practice of Advertising, 5th edition. Oxford : Elsevier, 268-280.

BARATS, Christine. 2009. «Textes numériques ou sur le numérique. Rhétorique de l'internationalisation et 'attractivité' des universités parisiennes ». In DEFAYS, J.-M. \& A. ENGLEBERT (dir.), Acteurs et contextes des discours universitaires, vol. 2. Paris : L'Harmattan, 209-225.

BARROW, Simon \& Richard MOSLEY. 2005. The Employer Brand, Bringing the best of brand management to people at work. Hoboken, NJ : John Wiley \& Sons.

BBC. 2017. « NHS staff shortages: Why so persistent? », 19 janvier 2017.

BBC. 2018a. «NHS vacancies a 'national emergency' », 11 septembre 2018.

BBC. 2018b. « NHS ‘haemorrhaging' nurses as 33,000 leave each year », 17 janvier 2018.

BBC. 2018c. « NHS ‘dangerously’ short of 100,000 staff », 21 février 2018 
BAUDET, Céline. 2003. « Le rédacteur et la fabrication du sens d'un texte persuasif ». Communication $22 / 2,44-62$.

BELTRAN-VIDAL, Danièle. 2007. « Les difficultés de traduction des mots de la santé dans l'espace européen ». Hermès La Revue 3/49, 83-87.

BENEDETTO, Caroline. 2017. « Contribution à l'étude de la fonction de régulation dans les domaines spécialisés anglophones : les professions à accès régulé au Royaume-Uni ». Thèse de doctorat, Université de Bordeaux.

BHATIA, Vijay. 2005. « Generic patterns in promotional discourse ». In HALMARI, H. \& T. VIRTANEN (dir.), Persuasion Across Genres: A Linguistic Approach. Amsterdam : John Benjamins Publishing, 213228.

BMJ (British Medical Journal). 2018. « BMA urges new health secretary to 'get a grip' on staffing crisis as vacancies increase ", 27 juillet 2018.

BOENISCH, Gilles. 2013. « Marie-Hélène Bacqué, Carole Biewener, L'Empowerment, une pratique émancipatrice?». Questions de communication 27, 386-387.

CARNET, Didier, Jean-Pierre CHARPY, Philip BASTABLE \& Philippe DELAGRANGE. 2017. L'Anglais médical à la Faculté. Paris : Ellipses.

CHARAUDEAU, Patrick. 2009. " Le discours de manipulation entre persuasion et influence sociale ", Acte du colloque de Lyon, 2009, consulté le 8 janvier 2019 <http://www.patrick-charaudeau.com/ IMG/pdf/Discours_Manipulation-_Texte_Lyon_.pdf>.

CHERNATONY (de), Leslie. 1999. « Brand management through narrowing the gap between brand identity and brand reputation ». Journal of Marketing Management 15/1-3, 157-180.

CHISHOLM, Alison \& Janet ASKHAM. 2006. A Review of Professional Codes and Standards for Doctors in the UK, USA and Canada. Oxford : The Picker Institute Europe.

DELL, David \& Nathan AINSPAN. 2001. Engaging Employees Through Your Brand. Conference Board, Washington D.C.

Department for Education. 2018. « Every Lesson Shapes a Life », $1^{\text {er }}$ octobre 2018, consulté le 20 décembre 2018 <https://getintoteaching.education.gov.uk\&gt>.

FAIRCLOUGH, Norman. 1995. Media Discourse. Londres : Edward Arnold.

FAURE, Pascaline. 2010. « Des discours de la médecine multiples et variés à la langue médicale unique et universelle ». ASp 58, 73-86.

FOWLER, Roger. 1991. Language in the News. Discourse and Ideology in the Press. Londres \& New York: Routledge.

General Medical Council (GMC). 2017. The State of Medical Education and Practice in the UK Report: 2017. Londres : GMC.

GIRARDEAU, Sophie. 2018. « Marque employeur : l'attractivité, une question d'expérience plus que de valeurs? ». Les Échos, 21 février 2018.

GOVONI, Norman. 2004. The Dictionary of Marketing Communications. Londres : Sage Publishing, Business and Economics.

HOEK, Leo Huib. 1981. La marque du titre. Dispositifs sémiotiques d'une pratique textuelle. Paris \& New York : De Gruyter Mouton. 
ISANI, Shaeda. 2001. «Calques et emprunts culturels ou le paradoxe de la culture professionnelle cible comme vecteur de perceptions erronées dans la culture professionnelle source - le cinéma et les professions juridiques ». Culture et communication en milieu professionnel interculturel. Université de Grenoble, 105-120.

KAPOOR, Vikram. 2010. «Employer branding: A study of its relevance in India ». Journal of Brand Management 7/1-2, 51-75.

KIEFER, Brittaney. 2018. " How the NHS tapped into national pride to recruit the next generation of nurses ». Campaign, 31 juillet 2018, consulté le 10 décembre 2018 <www.campaignlive.co.uk/ article/nhs-tapped-national-pride-recruit-next-generation-nurses/1488495>.

LACKA-BADURA, Jolanta. 2017. « Power relations between employers and potential employees reflected in the grammar of job advertising discourse ». Socjolingwistyka 31, 39-50.

LAUGIER, Sandra, Pascale MOLINIER \& Patricia PAPERMAN (dir.). 2009. « Qu'est-ce que le care? ». Souci des autres, sensibilité, responsabilité. Paris : Payot.

Law Society. 2018. " Get involved in the 2019 Solicitor Brand Campaign - we need your help! ». Londres : Law Society, consulté le 15 décembre 2018 <www.lawsociety.org.uk/policy-campaigns/ campaigns/solicitor-brand-campaign>.

LEHMANN, Denis. 1993. Objectifs spécifiques en langues étrangères. Paris : Hachette.

LLOYD, Stephen. 2002. « Branding from the inside out ». Business Review Weekly 24, 64-66.

LUGRIN, Gilles. 2006. Généricité et intertextualité dans le discours publicitaire de presse écrite. Berne :

Peter Lang.

MARTIN, Claude. 2008. «Qu'est-ce que le social care? Une revue de questions ». Revue Française de Socio-Économie 2/2, 27-42.

MILLER, William \& ROLLNICK, Stephen. 2013. L'entretien motivationnel - Aider la personne à engager le changement, traduit par P. Lecallier et D. Michaud. Paris : InterÉditions 12.

NHS Employers. 2014. « Running an effective campaign ». Londres : NHS Employers.

NHS Employers. 2018. « Inspire, attract and recruit - An interactive toolkit to support your workforce supply ». Londres : NHS Employers.

OGER, Claire \& Caroline OLLIVIER-YANNIV. 2006. « Conjurer le désordre discursif. Les procédés de 'lissage' dans la fabrication du discours institutionnel ». Mots. Les langages du politique 81, consulté le 29 mai 2018 <www.journals.openedition.org/mots/675>.

PETIT, Michel. 1999. « La fiction à substrat professionnel : une autre voie d'accès à l'anglais de spécialité ». ASp 23-26, 57-81.

PETIT, Michel. 2004. «Quelques réflexions sur la fiction à substrat professionnel : du général au particulier ». In PETIT, M. \& S. ISANI (dir.). Aspects de la fiction à substrat professionnel, Bordeaux : Université de Bordeaux, collection Travaux 2025, 3-23.

POWELL, Laura. 2014. « Glorification of the military in popular culture and the media ». In FORTE, M. (dir.), Good Intentions: Norms and practices of imperial humanitarianism. Montréal, Québec, Canada, 167-184.

PRATT, Siofra. 2016. « 7 of the best places to promote your employer brand ", 15 décembre 2017, Social Talent, consulté le 20 octobre 2018 <www.socialtalent.com/blog/recruitment/7-best-placespromote-employerbrand>. 
PRICE, Alan. 2007. Human Resource Management in a Business Context. Londres : Thomson Business Press.

RAO, Hayagreeva, Henrich GREVE \& Gerald DAVIS. 2001. « Fool's gold: social proof in the initiation and abandonment of coverage by Wall Street analysts ». Administrative Science Quarterly 46, 502526.

RIOU, Nicolas. 1999. Pub fiction : société postmoderne et nouvelles tendances publicitaires. Paris :

Éditions d'Organisation.

ROBERTS, Gareth. 1997. Recruitment and Selection: A Competency Approach. Londres : The Chartered Institute of Personnel and Development.

Royal Academy of Engineering (RAE) ET AL. 2018. «This is Engineering ». Londres : RAE, consulté le 29 mai $2018<w w w$. raeng.org.uk/education/this-is-engineering>.

Royal College of Nursing (RCN). 2018. « NHS reveals staffing crisis in new recruitment figures », 23 janvier 2018. Londres : RCN.

RYAN, Gerard, Marcel GUBERN \& Inma RODRIGUEZ-ARDURA. 2000. « Recruitment advertising: The marketing-human resource interface ». International Advances in Economic Research 6, 354-364.

SACRISTE, Valérie. 2001. « Sociologie de la communication publicitaire ». L’Année sociologique 51, 487-498.

SALMON, Christian. 2007. Storytelling. La machine à fabriquer les images et à formater les esprits. Paris : La Découverte.

SKELTON, John, Andy WEARN \& Richard новв. 2002. « 'I' and 'we': a concordancing analysis of how doctors and patients use first person pronouns in primary care consultations ». Family Practice 19/5, Oxford University Press, 484-488.

SLOWTHER, A. et al. 2012. « Experiences of non-UK-qualified doctors working within the UK regulatory framework: a qualitative study ». Journal of the Royal Society of Medicine 105/4, 157-165. SOULAGES, Jean-Claude. 2013. « La publicité à la télévision et les fictions de l'ordinaire ». Communication 32/1. DOI 10.4000/communicationorganisation.5375.

STUART, Toby, E. 2000. «Interorganizational alliances and the performance of firms: A study of growth and innovation rates in a high-technology industry ». Strategic Management Journal 21, 791811.

SWALES, John. 1990. Genre Analysis: English in academic and research settings. Cambridge : Cambridge University Press.

The Guardian. 2018. « NHS in England facing deepening staffing crisis, figures show ». 26 juillet 2018, consulté le 20 janvier 2018 <https://www.theguardian.com/society /2018/jul/26/nhs-inengland-facing-deepening-staffing-crisis-figures-show>.

TISSERON, Serge. 2011. «Intimité et extimité ». Communications 88, 83-91.

UK Government. 2017. « NHS workforce statistics June 2017 ». Consulté le 29 mai <www.gov.uk/ government/statistics/nhs-workforce-statistics-june-2017>.

UK Government. 2018. «Immigration rules Appendix K. Shortage occupation list ». Consulté le 29 mai <www.gov.uk/guidance/immigration-rules/immigration-rules-appendix-k-shortageoccupation-list>. 
VIOT, Catherine \& Laïla BENRAïss-NOAILLES. 2014. « Employeurs démarquez-vous ! La marque employeur, un gisement de valeur inexploité ? ». Management international 18/3, 60-81.

WÆRAAS, Arild. 2010. «Communicating identity: The use of core value statements in regulative institutions ». Administration \& Society 42/5, 526-549.

\section{ANNEXES}

\section{Annexe 1. Classification des films de recrutement intégrés au corpus}

\begin{tabular}{|c|c|c|}
\hline $\begin{array}{l}\text { Profession ou } \\
\text { spécialité }\end{array}$ & $\begin{array}{l}\text { Référence abrégée dans l'article, titre du film publicitaire, } \\
\text { nom de l'organisation de santé et adresse URL }\end{array}$ & $\begin{array}{l}\text { Date de mise en } \\
\text { ligne sur } \\
\text { internet }\end{array}$ \\
\hline \multirow[t]{2}{*}{$\begin{array}{l}\text { Médecine } \\
\text { généraliste } \\
\text { (General } \\
\text { Medicine) }\end{array}$} & $\begin{array}{l}\text { GM1. «A Career in General Practice ", Health Careers } \\
\text { <www.healthcareers.nhs.uk/explore-roles/doctors/roles- } \\
\text { doctors/general-practice-gp> }\end{array}$ & $12 / 06 / 2017$ \\
\hline & $\begin{array}{l}\text { GM2. "One Career, Endless Opportunities - \#choose GP ", } \\
\text { Health Education England } \\
\text { <www.youtube.com/watch?v=DQArWG9bGhc> }\end{array}$ & $19 / 10 / 2017$ \\
\hline \multirow[t]{3}{*}{$\begin{array}{l}\text { Psychiatrie } \\
\text { (Psychiatry) }\end{array}$} & $\begin{array}{l}\text { P3. «A Career in Psychiatry », NHS Medical Careers } \\
\text { <www.youtube.com/watch?v=9UhcfcC56AA> }\end{array}$ & 01/10/2012 \\
\hline & $\begin{array}{l}\text { P4. «A Career in Psychiatry », Health Careers } \\
\text { <www.healthcareers.nhs.uk/explore-roles/doctors/roles- } \\
\text { doctors/psychiatry> }\end{array}$ & 08/08/2016 \\
\hline & $\begin{array}{l}\text { P5. "Choose Psychiatry | A Career that Makes A Difference", } \\
\text { Royal College of Psychiatrists <www.rcpsych.ac.uk/ } \\
\text { discoverpsychiatry/acareerin } \\
\text { choosepsychiatry.aspx?theme=mobile> }\end{array}$ & $08 / 10 / 2018$ \\
\hline \multirow[t]{4}{*}{$\begin{array}{l}\text { Infirmerie } \\
\text { (Nursing, } \\
\text { Health Visiting) }\end{array}$} & $\begin{array}{l}\text { N6. "A day in the life of a Health Visitor ", NHS East of } \\
\text { England } \\
<\text { www.youtube.com/watch?v=5gz7X-AEUUw> }\end{array}$ & $22 / 07 / 2011$ \\
\hline & $\begin{array}{l}\text { N7. « Health Visitor Recruitment Campaign », NHS Midlands } \\
\text { \& East } \\
\text { <www.youtube.com/watch?v=XnD6ZYE0qJI> }\end{array}$ & $24 / 01 / 2012$ \\
\hline & $\begin{array}{l}\text { N8. « This is Modern Nursing ", Royal College of Nursing } \\
<\text { www.youtube.com/watch?v=KNIXlU47HQM> }\end{array}$ & $24 / 11 / 2016$ \\
\hline & $\begin{array}{l}\text { N9. «NHS Nursing Recruitment Video », Bolton NHS } \\
\text { Foundation Trust } \\
\text { <www.youtube.com/watch?v=HUzpLqnuRT4> }\end{array}$ & $12 / 05 / 2017$ \\
\hline
\end{tabular}




\begin{tabular}{|l|l|l|}
\hline & $\begin{array}{l}\text { N10. «NHS Wales' Nurse Recruitment Campaign », NHS Wales } \\
\text { <www.youtube.com/watch?v=QaOBLA8o-kI> }\end{array}$ & $12 / 05 / 2017$ \\
\hline & $\begin{array}{l}\text { N11. « We are the NHS - Nursing recruitment campaign », } \\
\text { Health Careers } \\
\text { <www.healthcareers.nhs.uk/working-health/we-are-nhs }\end{array}$ & $03 / 07 / 2018$ \\
\hline
\end{tabular}

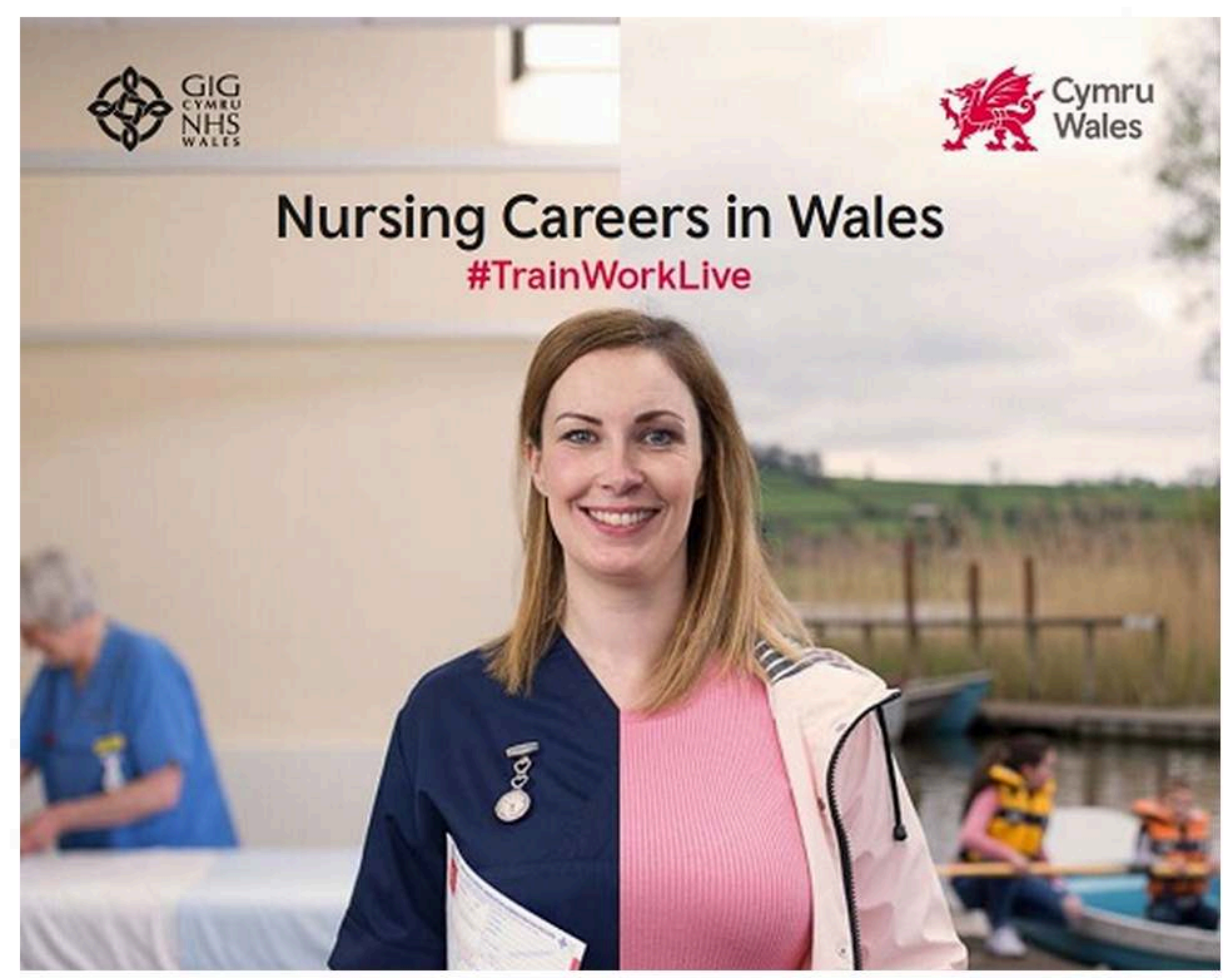

Annexe 2. Visuel extrait de la brochure « Nursing Careers in Wales » (N9)

Source : <https://trainworklive.wales>

Annexes 3 et 4 . Captures d'écran de quelques extraits de films qui donnent une impression de familiarité et de proximité 

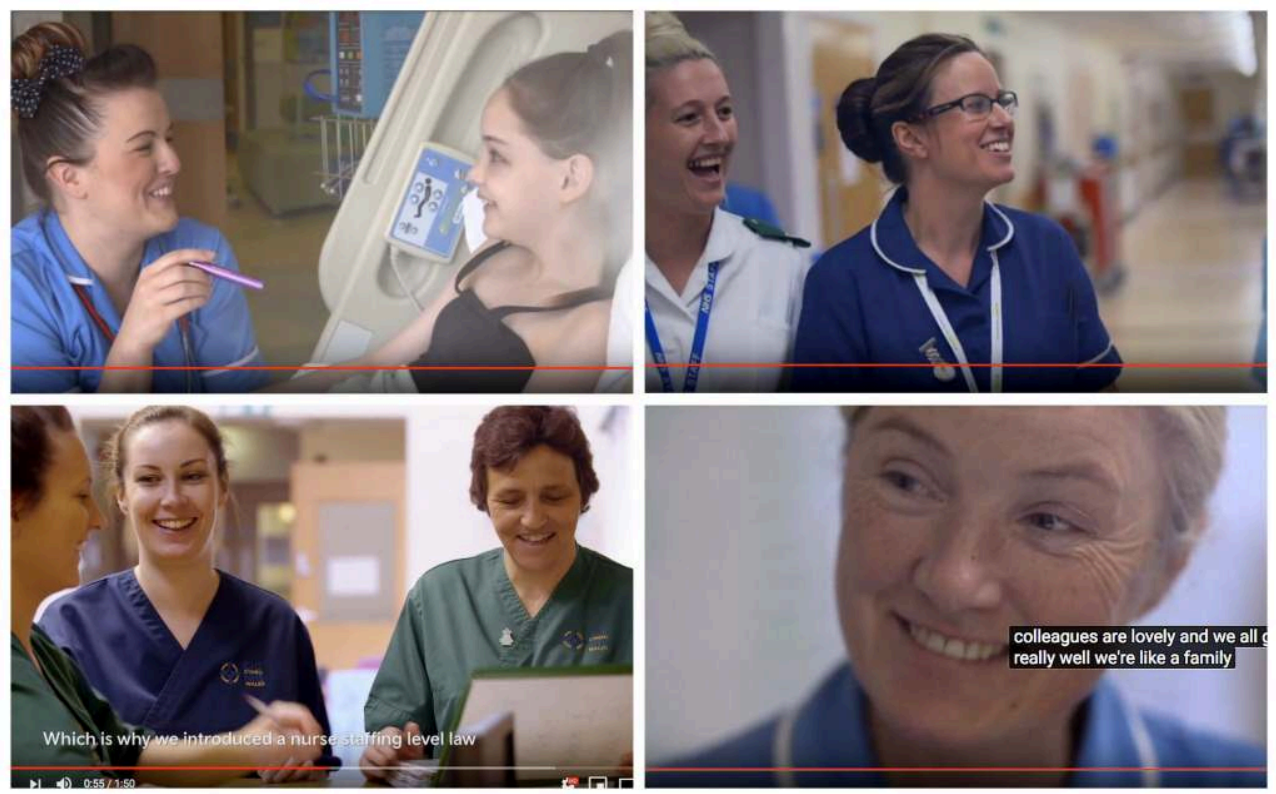

Annexe 3 «NHS Nursing Recruitment Video », Bolton NHS Foundation (N9)

Trust <www.youtube.com/watch?v=HUzpLqnuRT4>

Annexe $4:$ :We are the NHS - Nursing recruitment campaign », Health Careers (N11) <www.healthcareers.nhs.uk/working-health/we-are-nhs>

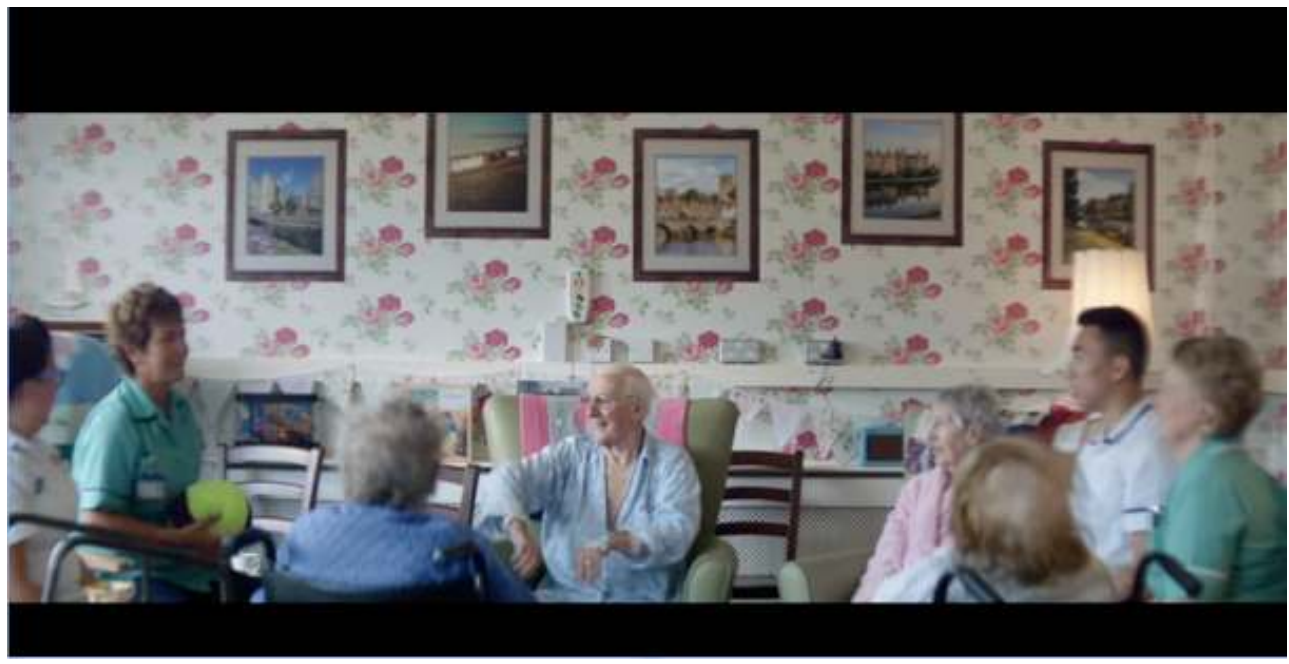

Annexe 5. Captures d'écran d'un extrait de film montrant la porosité des frontières entre les espaces personnel et professionnel

"We are the NHS - Nursing recruitment campaign », Health Careers (N11) $<$ www.healthcareers.nhs.uk/working-health/we-are-nhs> 


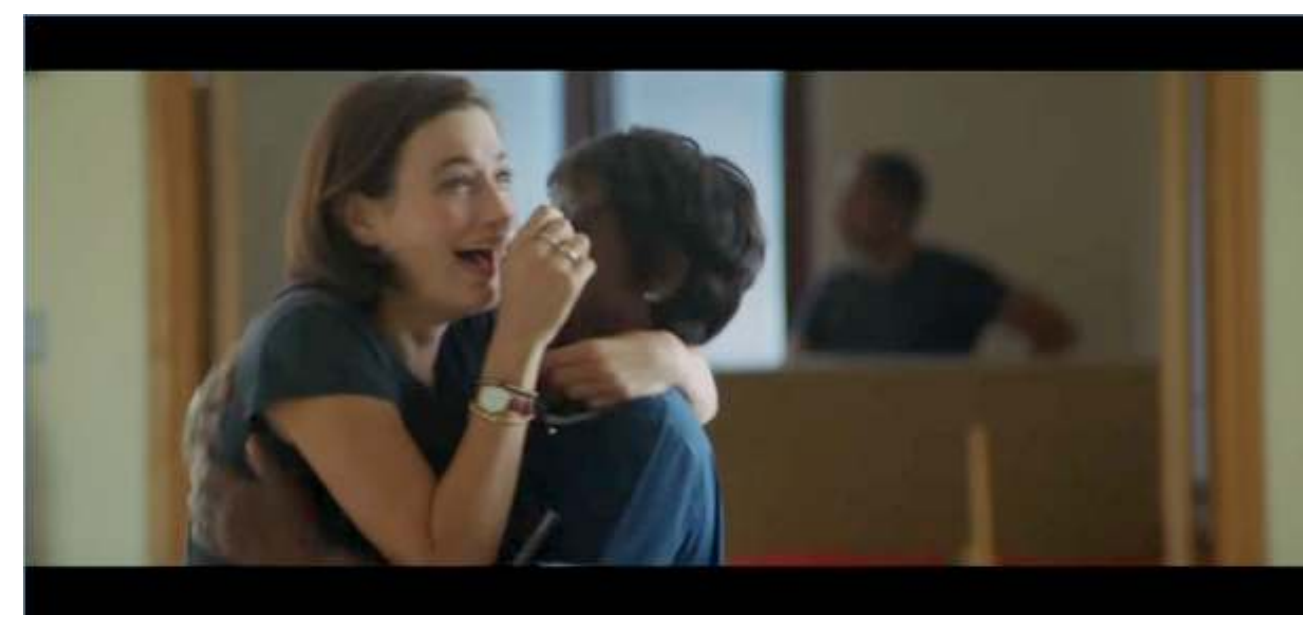

\section{NOTES}

1. Parmi les nombreuses définitions existantes, la conception de genre discursif que nous adoptons est celle de J. Swales (1990), qui met en avant la visée pragmatique de ces formes de communication, partagées et façonnées par une même "communauté de discours " dont les membres poursuivent des objectifs communs : « a genre comprises a class of communicative events, the members of which share some set of communicative purposes. These purposes are recognized by the expert members of the parent discourse community, and thereby constitute the rationale for the genre. This rationale shapes the schematic structure of the discourse and influences and constrains choice of content and style » (Swales $1990: 58$ ).

2. La structure concernée est alors amenée à communiquer sur elle-même, devenant à la fois sujet et objet de la communication.

3. Leur nom et l'intitulé exact de la fonction qu'ils occupent dans l'organisation sont d'ailleurs cités dans la plupart des films du corpus et peuvent être facilement vérifiés au moyen d'une recherche en ligne à partir du nom du professionnel.

4. Une recherche portant sur les définitions de ces termes et sur leurs dates d'apparition a été menée dans des dictionnaires spécialisés en ligne (AllBusiness.com, BusinessDictionary.com, Entrepreneur.com, notamment), ainsi que dans des revues professionnelles relevant du marketing (The Journal of Retailing, The Journal of Marketing, par exemple) et des ressources humaines (Strategic HR Review, McKinsey Quarterly).

5. En Angleterre, par exemple, l'organisation Health Education England a lancé les programmes Health Ambassadors et Inspiring the Future, qui font intervenir de façon ponctuelle et bénévole les étudiants en santé et les praticiens du domaine dans les établissements scolaires et universitaires pour informer le public sur le rôle des soignants et diffuser une image positive de leur métier.

6. Ces nominations ont été identifiées dans les établissements de santé suivants: Lambeth Commission Group, the Royal Devon and Exeter NHS Foundation Trust, University College London Hospital et Buckinghamshire Hospital.

7. Cette dynamique concerne autant les organisations que leurs collaborateurs, autrement dit les recruteurs comme les candidats, chacun étant désormais attentif à sa réputation.

8. Voir la page suivante: < www.gov.uk/government/statistics/nhs-workforce-statisticsjune-2017>, consultée le 01/07/2018.

9. La liste des postes pour lesquels il existe une pénurie de professionnels au Royaume-Uni et en Écosse, et auxquels peuvent candidater les praticiens étrangers est disponible à l'adresse 
suivante : <www.gov.uk/guidance/immigration-rules/immigration-rules-appendix-k-shortageoccupation-list>. Consultée le 29 mai 2018.

10. Certains médecins ont du mal, par exemple, à appréhender les différentes facettes de la communication non verbale (attitudes corporelles, expressions faciales, contact visuel, etc.) (Slowther et al. 2012: 105). D'autres sont peu coutumiers de certains principes comme la centralité du patient dans la relation de soin ou la prise de décision partagée, fondée sur la participation conjointe du patient et du soignant (ibid.).

11. L'entretien motivationnel (motivational interviewing) est une "méthode de communication directive, centrée sur la personne, et visant au changement de comportement par l'exploration et la résolution de l'ambivalence" (Miller \& Rollnick 2013, cités par l'Association française de diffusion de l'entretien motivationnel, AFDEM). De nombreux praticiens du domaine de la santé y ont recours pour encourager leurs patients à faire preuve d'autonomie.

12. On peut citer les questions suivantes, tirées du corpus: "What attracted you to a career in health?» (GM1) ; "What makes psychiatry a particularly good career? And what sort of opportunities are there that you can highlight to sell this career? » (P3) ou encore " if you could describe your role in one word, what would it be?» (P4).

13. Cette affirmation vise aussi, nous semble-t-il, à convaincre les spectateurs de la réussite du modèle partenarial entre les divers acteurs du domaine de la santé, une forme de coordination activement promue par les organisations du NHS depuis le début des années 1990 (Benedetto $2017: 156-159)$.

14. Identifiée en premier lieu comme genre littéraire par M. Petit (1999), la fiction à substrat professionnel (FASP) réunit les codes du thriller, du roman policier, et les caractéristiques d'un milieu de travail donné, notamment ses pratiques, sa culture et sa langue. Désormais, elle ne se limite pas à la fiction écrite mais concerne aussi les productions cinématographiques et télévisuelles.

15. La culture professionnelle, telle que nous l'envisageons, est la connaissance de «principes plus ou moins clairement exprimés, en conduite du métier, accomplissement de la vocation, règles de conduite, normes de comportement, attitudes en réponse à des situations données " (Bernard 1990: 4 cité par Lehmann 1993: 19), autant de caractéristiques qui reflètent la philosophie et les valeurs qui sont mises en avant par les dirigeants d'une organisation.

16. Plusieurs énoncés du corpus font explicitement référence à la notion de care : « you can step up to care for them » (N8), "I care for the patients across their whole surgical pathway » (N8), " my patients need to be the centre of my care because it's not about me, it's about the patients " (N8), " to be able to care for these people who are such in a vulnerable position " (N9).

17. Ces métaphores mettent en avant les compétences techniques des soignants mais aussi et surtout leurs qualités relationnelles ("we are experts of the human body - and human emotion too", N11), notamment leur respect et leur bienveillance à l'égard des soignés (« we are a guest in the patient's home », N9), ainsi que l'impact du soin sur la vie des patients (« we're mending lives », N11). 18. Dans son acception anglaise, la notion de care combine « le care about et le care for someone, autrement dit le soin et l'affection» (Martin 2008: 28). Elle met donc en jeu aussi bien les compétences techniques que les compétences relationnelles des soignants. Sans réel équivalent français, elle est généralement traduite par un verbe d'action qui signifie "prendre soin ", "se soucier de " ou bien par un substantif qui peut être rendu, suivant les contextes, par "soin", « attention », « sollicitude », « concernement » (Laugier et al. 2009).

19. Dans le domaine de la santé, l'empowerment désigne la capacité des personnes ou de groupes de personnes à agir pour assurer leur bien-être. La notion articule deux dimensions, « celle du pouvoir, qui constitue la racine du mot, et celle du processus d'apprentissage pour y accéder » (Bacqué \& Biewener citées par Boenish 2013: 386). Plusieurs expressions françaises comme « capacitation », " puissance d'agir » ou " pouvoir d'action » ont été inventées pour retranscrire 
la notion mais elles ne rendent pas compte du processus d'apprentissage menant à l'émancipation des sujets ou de la dimension collective de la démarche (ibid.).

20. À titre d'illustration, nous reproduisons cet extrait du corpus qui concerne la profession infirmière : "our nursing structure is based on the 60s and the strapline that we put is about looking people's potential; supporting, encouraging and empowering people to do the right things for patients".

21. La notion de care est apparue aux États-Unis dans les années 1980 avant de devenir un objet d'étude multi-référentiel dans le monde anglophone. Celle d'empowerment est également originaire des États-Unis, où elle a été introduite dans les années 1960. Elles ne se sont répandues en France qu'au début des années 2000.

\section{RÉSUMÉS}

Cette contribution a pour objet la caractérisation d'un genre de discours professionnel : les films de recrutement produits par les organisations de santé britanniques. Ces productions audiovisuelles visent non seulement à attirer de nouvelles recrues, mais aussi à projeter une image positive des organisations pour leur donner plus de crédibilité et de légitimité. En prenant appui sur un corpus de films de recrutement réalisés entre 2011 et 2018 dans le domaine de la santé, nous nous interrogeons sur les modes de représentation de l'identité professionnelle dans le discours. Quelle image les locuteurs donnent-ils à voir d'eux-mêmes et de leur profession, et quels procédés stylistiques et rhétoriques mobilisent-ils pour emporter l'adhésion de leur public ? L'article se clôt par une réflexion sur l'intérêt que présente le film de recrutement pour l'enseignement de l'anglais de spécialité dans sa dimension culturelle.

This paper aims at contributing to the characterisation of a professional genre of discourse: the recruitment films of British health organisations. These audiovisual productions are used not only to attract new employees, but also to convey a positive image of organizations to give them more credibility and legitimacy. Drawing on a corpus of recruitment films produced between 2011 and 2018 in the health sector, we examine how professional identity is being represented in the discourse. What image do the speakers give of themselves and their profession, and what stylistic and rhetorical strategies do they use to convince their audience? Lastly, we examine the extent to which recruitment films can be of interest to ESP teaching in its cultural dimension.

\section{INDEX}

Mots-clés : culture professionnelle, domaine de la santé britannique, film de recrutement, publicité institutionnelle, vulgarisation

Keywords : institutional advertising, popularisation, recruitment film, UK health sector, work culture 


\section{AUTEUR}

\section{CAROLINE BENEDETTO}

Caroline Benedetto enseigne l'anglais des affaires et la communication professionnelle à

l'Université Paris Descartes, au sein de la filière commerciale de l'IUT. Docteur en anglais, elle s'intéresse à différents aspects de la langue de spécialité : analyse stylistique et rhétorique des genres de discours spécialisés, terminologie et néologie, culture des domaines spécialisés.

caroline.benedetto2@gmail.com 